\title{
The technologies of application and design features of layer by layer milling machines
}

\author{
Anton Cheban ${ }^{1 *}$ and Natalia Khrunina ${ }^{1}$ \\ ${ }^{1}$ Mining Institute of Far eastern branch of Russian Academy of Sciences, Khabarovsk, Russia
}

\begin{abstract}
Significant quantity of surface miners, various designs and engineering factors are involved in open-cut mining. The article authors offer expanded classification of applied and perspective surface miners with regard to features of design of work tools, rock destruction tool, undercarriage and other elements. Key words: surface miner, massif destruction, picks, mills, undercarriage, layer by layer mining
\end{abstract}

\section{Introduction}

The technology of blastless mining of solid mineral deposits with the application of surface miners become more common in various world countries [1-3]. Surface miners mine the deposits of coal, bauxites, phosphorites, marls, gypsum, limestones and other minerals. The degree of the application of mineral deposits blastless mining technologies expand embracing new deposits built up with increasingly more coherent rocks. The transfer of enterprises to new technologies is conditioned by both economical factors and material enhancement of measures of ecological and social safety of construction and mining which are limited by drilling and blasting operations.

Loosening of massif using a blast has a row of disadvantages. In case if work front approaching close to inhabited localities or infrastructure facilities, drilling and blasting operations is completely excluded, at the same time, in a row of long operated deposits, basic and more quality reserves (for the reason of mining of other reserves) of minerals are found to be in the immediate proximity to residential space and other facilities.

Currently, the production of surface miners is performed by a great number of companies producing equipment for mining and construction, surface miners by Wirtgen, Man Takraf and Krupp companies got widespread. Active search for optimal engineering and layout designs in order to ensure high performance and reliability of machinery is conducted with the creation of everything new in mechanical engineering. As a result, by now, great number of miners significantly differing from one another by design, scope of application and operating principle has been created [2-3]. In order to ensure effective interaction of surface miners and other types of mining machinery operating together with them, it is necessary to optimize basic parameters by which the selection of equipment is made, to that end authors have made an attempt to classify known designs of surface miners as well as features of their work equipment and undercarriage.

* Corresponding author: chebanay@,mail.ru 


\section{Scope of application and design features of surface miners}

Layer by layer which got widespread, and benching technologies (fig. 1) may be distinguished by the technology of deposits mining with surface miners. In case of layer by layer mining technology, miner travels along pit face with rather high speed, surface miners designed for layer by layer mining of deposits are produced by such companies as Wirtgen, Man Takraf, Krupp and others. Such miners are simpler by design, more reliable, more compact and cheaper than machinery for mining of deposits by benching, and also guarantee more effective selective mining of mineral in case of mining of flat and shallowdipping beds [3-5]. Miners performing mining of deposits by benching have gained lesser spread, they are produced by such companies as Alpine Westfalia, Eickhoff, "MGGUGeofrez" and others. Work tool of such miners is located on a boom therefore these miners are called boom ones which allows mining a deposit by benches with the height of up to 10 $\mathrm{m}$. In case of benching, surface miner travels across pit face manifold slower compared to machinery operating by layer by layer mining technology, due to this a row of advantages appears, namely: in order to ensure high-performance operation of a surface miner there is no need in creation of extraction front of significant extent; the integration of surface miners into mining process diagram becomes simpler which guaranties production flow; mineral mining selectivity quality improves with steeply dipping bed mining; miner connection to external power source becomes possible which reduces operational costs of a miner operation and increases ecological cleanliness of production.

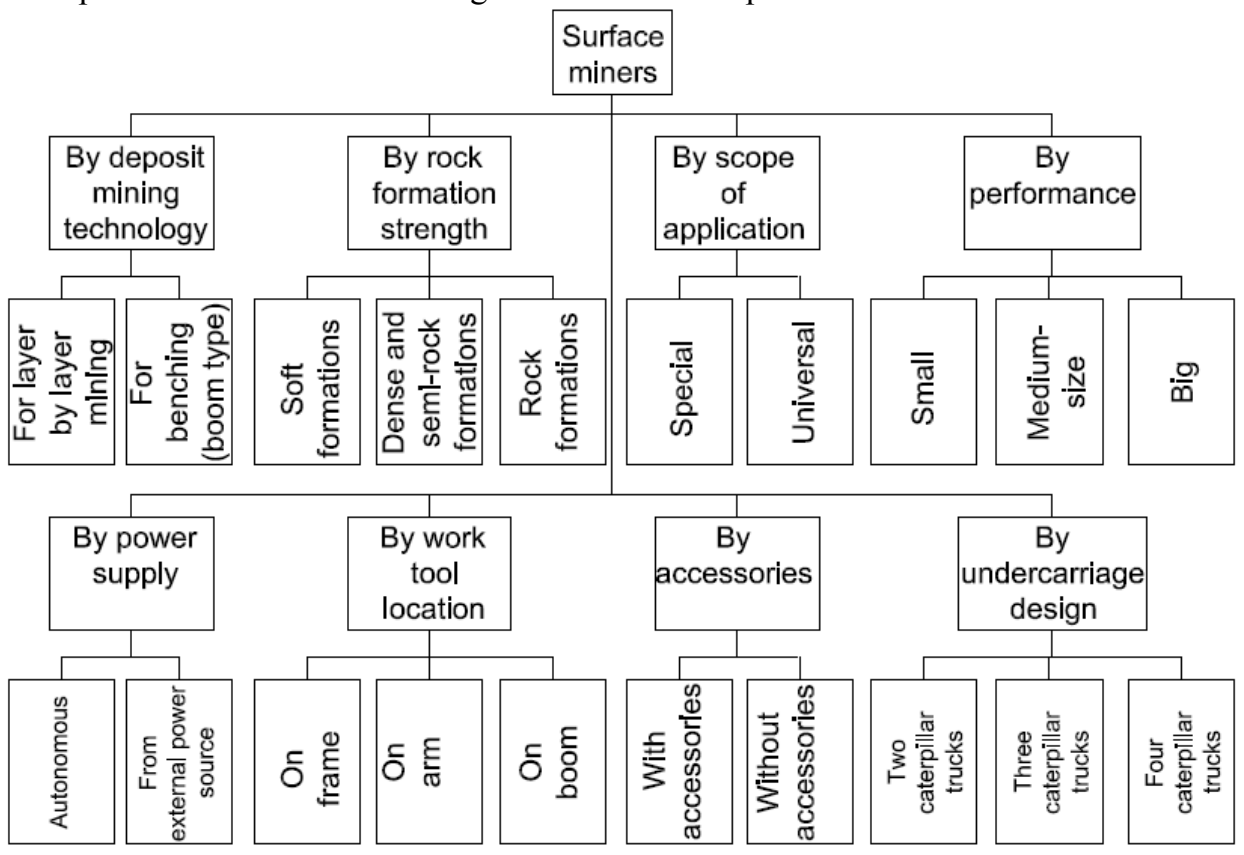

Fig. 1. Peculiarities of design and methods of surface miners operation

Surface miners are designed for the mining of a certain class of rock formations. First surface miners - excavating and milling machines appeared in the mid of the previous century were designed to mine soft formations: sand clays, loams, clays, etc., these machines turned out to be very effective at the construction of trunk lines, reservoirs and other large facilities. In 80's of the previous century, surface miners for blastless selective mining of dense (soft and medium-hard coals, marl, gypsum and others) and semi-rock (phosphorites, bauxites, limestones, shales, hard coals and others) formations [4, 6]. These 
machines successfully compete with bucket-wheel excavators, especially in case of the development of complex structure mineral deposits. In connection with the expansion of technologies of layer by layer mining in mining operations, by now, surface miners for effective extraction of easily mined rocks (with compressive strength of 50-80 MPa and more), for example, of limestones being row materials for cement production, have been created [4].

One of prototypes of modern surface miners along with excavating and milling machines was road milling machine designed for layer by layer destruction and scraping when repairing motor roads and aerodromes. Big road milling machines were advantageously operated in case of the development of complex structure deposits built-up with dense and semi-rock formations [6]. Consequently, surface miners may be subdivided by purpose into special, usually medium-size and large machines designed for overburden and mining operations in pits, and universal, as a rule small miners or big road milling machines which may be deployed both in mining operations and construction. It should be noted that big surface miners may be either used at especially large construction works [5].

Manufacturing companies produce various technical parameters of made miners (performance is given in $\mathrm{m}^{3} / \mathrm{h}$ in dense mass, in $\mathrm{m}^{3} / \mathrm{h}$ of excavated rock, $\mathrm{t} / \mathrm{h}$ of excavated rock), and so far there is no shared vision of what is the main parameter of a miner, for example, if we judge by surface miner tagging, for machines by Wirtgen company - this will be a width of strip being milled in $\mathrm{mm}$, for miners by Man Takraf company - rated capacity in solid in $\mathrm{m}^{3} / \mathrm{h}$, and for miners by Krupp - theoretical capacity by loosen rock in $\mathrm{m}^{3} / \mathrm{h}$ [7-8]. With regard to national practice for the classification of bucket-wheel excavatorsby their rated technical performance for loosen mass, it is possible to accept given gradation as well as for surface miners within the limits as follows: small - to 630 $\mathrm{m}^{3} / \mathrm{h}$, medium-size - to $2500 \mathrm{~m}^{3} / \mathrm{h}$ and big - over $2500 \mathrm{~m}^{3} / \mathrm{h}[8]$.

Miners operating by layer by layer technology are actuated by diesel engine, this enables operation in autonomous mode irrespective of the availability of electrical networks in the area of mining operations which is especially important in areas where there are no centralized electrical networks and power is generated by means of diesel power plants. At that, a miner may easily move along an open-cut in case if it is necessary to operate at a new site. Engines of miners operating by benching, are basically actuated by external power sources.

Miners design maps provide for various variants of a work tool location. As much, surface miners by Man Takraf (model MTS) and Wirtgen (model SM) have a work tool mounted on a frame, accordingly, in a front and mid parts of the machine [5]. Miners by Krupp (model KSM) have a work tool mounted on an arm. All the afore-said models of miners are designed for layer by layer deposit mining technology. Surface miners developing a deposit by benching has a work tool mounted on a boom, such machines are produced by companies Alpine Westfalia (models VASM), "MGGU-Geofrez" (model KGF-80) and others.

Miners may be outfitted with accessories, for example, with bed boundary detection system which allows selective extraction of mineral and barren rock in automatic mode, some miners are equipped with a special shakers for separation of obtained mineral matter immediately in an open-cut [5].

Undercarriage of surface miners may consist of two, three or four caterpillar trucks. Two-caterpillar truck undercarriage ensures maximum mobility of a miner which is rather critical when operating at areas short in length. Design with three or four caterpillar trucks is used provided that a work tool is located in the central part of a miner, swing of such a miner is made by means of caterpillar trucks turning around hydraulic racks which these trucks are hung on [5]. 


\section{Analysis of designs of surface miners work tools}

By design, work tools of surface miners may be divided into three groups. Screw and milling work tools representing screws and drums, on which rock destruction tool is mounted, got widespread, they allow mining dense, semi-rock and rock formations [4]. Bucket work tools represented by bucket-wheels installed on machines type KSM by Krupp, ZFM type by Voronezh Plant and by some other companies may excavate soft, dense and semi-rock formations. Plough work tools gained limited application and mounted on machines designed for the development of soft and some dense formations (fig. 2).

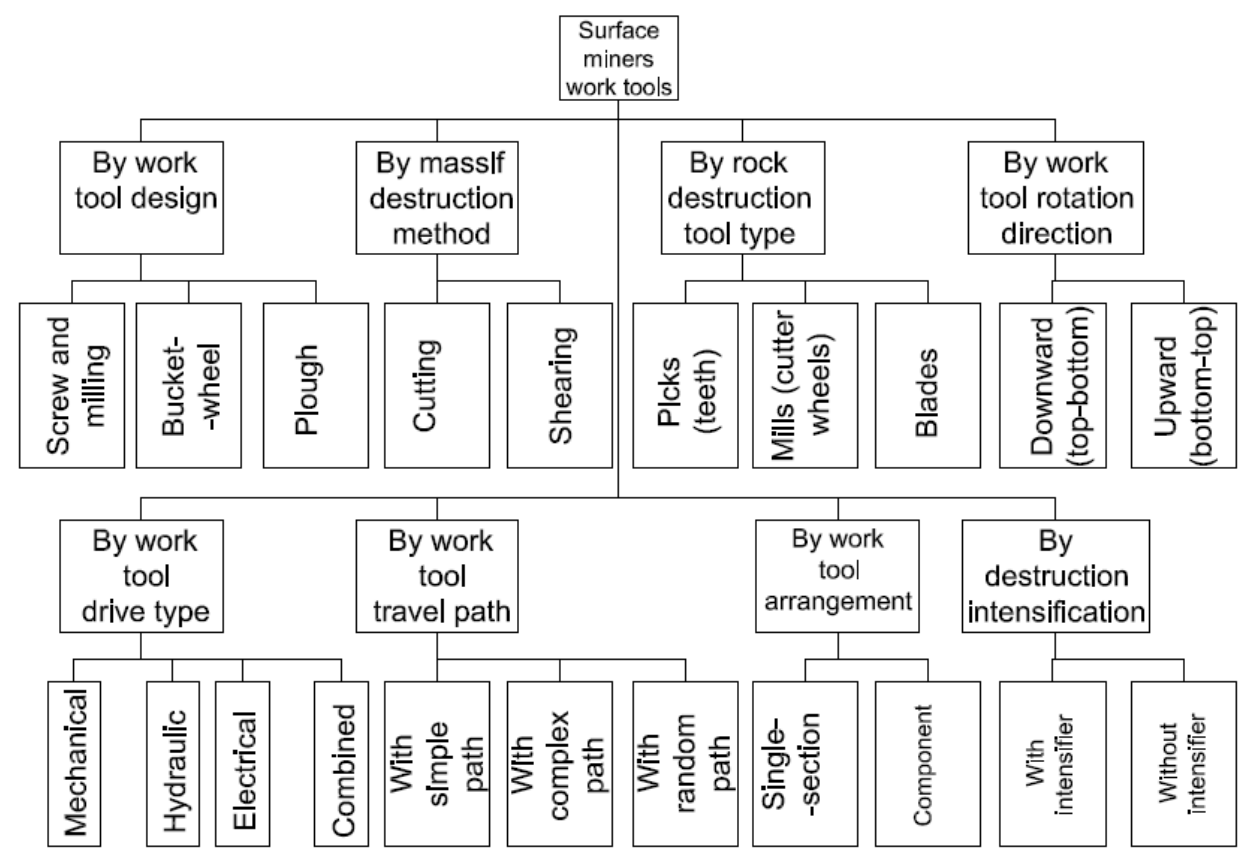

Fig. 2. Surface miners work tools design peculiarities

The destruction of formations with a surface miner is carried out via cutting or shearing massif using a rock destruction tools installed on a work tool. The destruction via cutting is applied in case of the development of soft and some dense formations and destruction by shear - dense, semi-rock and rock formations.

Picks (teeth), mills (cutter wheels) and blades may be used as rock destruction tool. Picks (teeth) gained utmost spread, they are installed on screw and milling and bucketwheel work tools [5]. Mills (cutter wheels) and blades on surface miners gained limited application and are installed, correspondingly, on screw and milling and plough work tools.

Depending on the direction of work tool rotation, picks penetrate into formation from top to bottom (downward mining) or from bottom to top (upward mining). In case of downward mining, the contact between loose rock and a work tool drum is minimized resulting in the reduction of wear of picks and pick boxes, decrease in the intensity of adhesion of wet stock onto a work tool as well as the reduction of possibility of large rock blocks generation as a results of breaking of oversized flagstones in case of the mining of robust formations of stratified structure.

A surface miner work tool drive may be mechanical, electrical or combined (electrohydraulic) one. Torque of a diesel engine, hydraulic actuator or electric motor is transferred using belt or chain transmission. Promising trends are direct drive of a work tool when hydraulic or electric motor is built inside a work tool. Normally, a surface miner work 
tool has one common drive, however component work tools may be equipped with several drives (each of cutting units has a separate drive unit and a transmission).

Depending on the complexity of suspension, a surface miner work tool may travel by various paths. In case of a simple travel path, a work tool moves up and down ascending into transport position and descending into operating position. Complex travel path is provided by a miner work tool moving up and down and its inclination in against a frame in vertical plane. Functional capability of a work tool inclination enables forming flat even work site of an open-cut with a slope towards settling sump for water drainage. Random travel path performed in designs of boom miners enables a work tool to travel freely and in any direction in horizontal and vertical planes, ascend, descend, turn in plan, telescope a boom which makes deep selectivity of mineral mining possible $[3,5]$.

By arrangement, a surface miner work tool is often a single-section one representing screw or drum on which rock destruction tools are mounted as per a certain scheme. A work tool also may consist of two and more identical cutting units as with miners by Alpine Westfalia company (model VASM) [5].

In order to increase efficiency of rock formations destruction process, a work tool may be outfitted with destruction process intensifiers, for example, ones that ensure vibrational movement of a work tool or the capability of its reciprocating offset (in relation to a miner boom), active rotor work tool design equipped with impact devices has been developed [5].

\section{Conclusions}

Surface miners are effective mining machinery which introduced into mining a great deal of innovative process solutions for mining of solid mineral deposits. Undoubtedly that manufacturing companies carry on improvement and development of designs and process parameters of surface miners and the scope of application of these machines at open-cut mining and in construction expands.

\section{References}

1. Wirtgen surface mining for selective limestone mining in the North Caucasus / Russia. Zement-Kalk-Gips Int. 67, 10, p. 18. (2014).

2. A.Iu. Cheban The application of milling machines in construction and mining or construction materials // Bulletin of Pacific national university. 3. pp 105-108. (2012).

3. E.E. Shvabenland Concerning the potential of continuous milling machines with the development deposits by open-cut mining // Rational subsoil development. 1. pp 54-60. (2014).

4. M. Pikhler, Ui.B. Pankevich Directions of development and results of the application of miners by Wirtgen Surface Miner at open-cuts and open-casts of the world // Mining industry. 3. pp 42-45. (2000).

5. A.R. Mattis and others Blastless technologies for open-cut mining of solid minerals. Novosibirsk: Publishing house of Siberian Branch of Russian Academy of Sciences, p. 337. (2007).

6. Iu.B. Pankevich, G. Khartman Experience of surface miners by company Wirtgen on plaster quarries of the world // Mining industry. 1. pp 4-9. (1997).

7. Drebenshtedt K., Pessler C. Calculation methods for mining machines. Materials of the international conference "Forum of miners - 2006". D: National mining University. pp. 2632. (2006).

8. K.N. Trubetskoi, M.G. Potapov, K.E. Vinitskii, N.N. Melnikov and others. Open-cut mining. Bureau of Mines. p. 590. (1994). 